\title{
Stabilization for a Class of New Chaotic Systems with Adaptive Quantizer
}

\author{
Y.H. Zhai \\ School of Automation, Guangdong University of \\ Technology, Guangzhou 510006, P.R. China \\ School of Information Engineering, Guangdong University \\ of Technology, Guangzhou 510006, P.R. China
}

\author{
Y.H. Wang \\ School of Automation, Guangdong University of \\ Technology, Guangzhou 510006, P.R. China
}

\begin{abstract}
This paper investigates the asymptotical stabilization via state feedback for a class of new chaotic systems, which nonlinear terms are monotonically increasing odd functions with the range $[-1,1]$, with a quantizer connected on the input channel. The updated law and adaptive law of estimate boundary error of quantizer are derived firstly. By the help of it, the nonlinear adaptive controller is proposed to ensure the chaotic system to be stabilized asymptotically. A simulation example is utilized to demonstrate the validity of the results in this paper.
\end{abstract}

Keywords-chaotic system; stabilization; adaptive quantizer; state quantization

\section{INTRODUCTION}

The stabilization of chaotic systems is important, for the unpredictable and irregular chaotic behaviours in engineering practice are always harm to the normal operation of the system.[1-7] The state feedback control methods are utilized to stabilizing Chaos.[8-12] The continuous state measurements are directly transferred to the feedback controller in these occasions. In fact, the system states usually need to be detected and transmitted via some additional information processing equipments such as sensors, encoders and digital transmission equipments.[13] It is well known that the hardware always possessing some form of imprecision and uncertainty. Therefore, if a digital device is used in the chaotic systems controlled, the finite number of quantized values of system states maybe causes instability.[14,15] Therefore, it is necessary to investigate the stabilization approaches for the chaotic systems via feedback control with quantized state measurements.

The feedback stabilizing systems with quantized states have been researched continuously in decades. The static quantization method was applied to the stabilization of linear systems in [10-14]. The time-varying quantizing feedback stabilizing controllers are designed for nonlinear systems in [16-18]. The results show that the time-varying quantizing methods can stabilizing nonlinear systems better than the static quantization approaches. Inspired by the corresponding results in [17-19], a new adaptive nonlinear controller is designed to feedback stabilize a class of new chaotic systems in which the nonlinear terms are monotonically increasing odd functions with the range $[-1$, $1]$.

The remainder of this paper is organized as follows. Sec.
2 gives dynamic models of chaotic systems. In Sec. 3, a nonlinear controller is designed for asymptotically stabilizing the chaotic systems, and the adaptive updated laws of quantizers and the controller are proposed. Sec. 4 shows the effectiveness of method in this paper by a numerical simulation illustration. The conclusions is given in Sec. 5.

\section{A CLASS OF NEW CHAOTIC SYSTEMS}

In paper [20], a class of new chaotic dynamical systems are described in the following nonlinear differential equations

$$
\dot{x}=A x+F(x)+C,
$$

where

$$
x=\left(\begin{array}{lll}
x_{1} & x_{2} & x_{3}
\end{array}\right)^{T}
$$

is

state

vector, $A=\left(\begin{array}{ccc}0 & 1 & 0 \\ 0 & 0 & 1 \\ -a & -a & -a\end{array}\right) ; F(x)=\left(\begin{array}{llll}0 & 0 & b f(x)\end{array}\right)^{T}$ and the nonlinear term $f(x)$ takes the form $f(x)=f_{i}\left(x_{1}\right)$, $i \in\{1,2, \ldots, 8\}$ in (2); $C$ is constant vector but equals zero in (1), the parameters $a \leq 1$ and $b$ are positive constants.

$$
\begin{gathered}
f_{1}(x)=\frac{1-\exp \left(-x_{1}\right)}{1+\exp \left(-x_{1}\right)}, \\
f_{2}(x)=\frac{2}{\pi} \arctan \left(\sinh \left(x_{1}\right)\right), \\
f_{3}(x)=\tanh \left(x_{1}\right), \\
f_{4}(x)=\frac{2}{\pi}\left(\frac{\tanh \left(x_{1}\right)}{\cosh \left(x_{1}\right)}+\arctan \left(\sinh \left(x_{1}\right)\right),\right. \\
f_{5}(x)=\frac{3}{2} \tanh \left(x_{1}\right)\left(1-\frac{\tanh \left(x_{1}\right)}{3}\right), \\
f_{6}(x)=\frac{2}{\pi} \arctan \left(x_{1}\right),
\end{gathered}
$$




$$
\begin{gathered}
f_{7}(x)=\left\{\begin{array}{l}
1, \quad x_{1} \geq \frac{1}{k}, \\
k x_{1},-\frac{1}{k} \leq x_{1}<\frac{1}{k}, k>0, \\
-1, x_{1}<-\frac{1}{k},
\end{array}\right. \\
f_{8}(x)=\left\{\begin{array}{l}
\frac{x_{1}^{n}}{x_{1}^{n}+1}, \quad x_{1} \geq 0, \\
-\frac{\left(-x_{1}\right)^{n}}{\left(-x_{1}\right)^{n}+1},
\end{array} \quad x_{1}<0,\right.
\end{gathered}
$$

It is clearly that the nonlinear functions $f(x)$ are monotonically increasing odd functions with the range $[-1$, 1]. It should be pointed out that the nonlinear term $f_{i}(x)$, $i=1,2,3,4,5$ are the activation functions of neural networks proposed in [20, 21]. Furthermore, the system (1) with $f(x)=f_{6}(x)$ corresponds to the chaotic system proposed in [22], and the system (1) with $f(x)=f_{7}(x)$ corresponds to the chaotic Chua's circuit system in [23]. Additionally, note that the new systems (1) proposed exhibit chaos mainly corresponding to the parameter $a \leq 1$. We assume the slope $k=1$ in $f_{7}(x)$ and only refer to $\mathrm{n}=1$ in $f_{8}(x)$ if not stated otherwise in the sequel, then we can derived that [21]

$$
|f(x)| \leq \frac{3}{2}\left|x_{1}\right| \leq \frac{3}{2}\|x\|_{2}, \forall x \in R .
$$

\section{TIME-VARYING QUANTIZER}

Generally speaking, a quantizer is defined as a piecewise function $q: R^{n} \rightarrow L, x \rightarrow q(x)$, where $L$ is a finite subset of the space $R^{n}$.[16,17] This leads to a partition of $R^{n}$ into a finite number of quantization regions of the form $\left\{x \in R^{n}: q(x)=l\right\}, l \in L$. The shapes of these quantization regions are arbitrary. When $z$ does not belongs to the union of quantization regions of finite size, the quantizer saturates.[16,17] More precisely, we assume that the quantizer satisfies the following two conditions:

$$
\begin{gathered}
\|q(x)-x\|<\varepsilon, \text { when }\|x\| \leq M, \\
\|q(x)\|>M-\varepsilon, \text { when }\|x\|>M,
\end{gathered}
$$

where $M$ is quantization range and $\varepsilon$ is quantization error bound. The condition (4a) gives the quantization error bound when the quantizer is unsaturated, while condition (4b) shows the detection of the saturation of quantizer. A typical quantizer is given as follow,

$$
q(x)=\left\{\begin{array}{l}
M \quad \text { when } \mathrm{x}>(\mathrm{M}+0.5) \varepsilon, \\
-M \quad \text { when } \mathrm{x} \leq-(\mathrm{M}+0.5) \varepsilon, \\
{[x / \varepsilon+0.5] \text { when }-(\mathrm{M}+0.5) \varepsilon \leq \mathrm{x} \leq(\mathrm{M}+0.5) \varepsilon,}
\end{array}\right.
$$

where $[x]$ is the floor function.[17]
By introducing a time-varying factor $\beta(t)>0$ into the quantizer $q(x)$, the time-varying quantizing feedback stabilizing controllers can be designed for nonlinear systems. Then the quantizer $q(x)$ is changed to follows

$$
\bar{q}=\beta q\left(\frac{x}{\beta}\right) .
$$

A typical example of these quantizers is a digital camera with fixed pixels and zoom lens, and the focal length of the lens is just the time-varying factor $1 / \beta$.

\section{STABILIZING THE NEW CHAOTIC SYSTEMS}

For stabilizing the chaotic systems, a nonlinear feedback controller can be designed, and the closed-loop systems are shown as below

$$
\dot{x}=A x+F(x)+C+u .
$$

If the states of systems (7) don't need to be quantified, i.e. without a quantizer in the input channel of controller, we can choose a nonlinear controller as

$$
u=K x-F(x)-C,
$$

where $K$ is a gain matrix, the closed-loop system is obtained as $\dot{x}=(A+K) x$. This implies that as long as the matrix $A+K$ is Hurwitz, the system (1) will be asymptotically stabilized. The gain matrix $K$ may be obtained by solving linear matrix inequality (LMI) as below,

$$
X A^{T}+A X+Y^{T}+Y<0,
$$

where $X>0$, and $K=Y X^{-1}$.

Assumption 1 Consider the controlled chaotic systems (7). The matrix $K$ is chosen so that $A+K$ is a Hurwitz matrix.

When the system states are quantized by the quantizer (6), the corresponding controller is proposed as follows.

$$
\begin{gathered}
u=K\left(\beta q\left(\frac{x}{\beta}\right)\right)-F\left(\beta q\left(\frac{x}{\beta}\right)\right)-C, \text { when }\|x\| \leq|\beta| M, \text { (10a) } \\
u=-C, \text { when }\|x\|>|\beta| M .
\end{gathered}
$$

where the matrix $K$ is chosen such that for a given positive definite matrix $Q$, the following Lyapunov equation has only one positive definite matrix solution $P$.

$$
(A+K)^{T} P+P(A+K)=-Q
$$

Remark 1 The matrices $K$ and $P$ in Lyapunov equation (11) can be obtained by solving the linear matrix inequality (LMI) $X A^{T}+A X+Y^{T}+Y<0$, where $X>0$, and $K=Y X^{-1}$.

The update law of $\beta(t)$ in controller (10) is proposed as follows, when $\|x\|>|\beta| M$ the update law is equation (12a), if $\|x\| \leq|\beta| M$ then get equation (12b). 


$$
\begin{aligned}
& \dot{\beta}(t)=\frac{1}{2 \beta M^{2}}\left[\lambda_{0}+\lambda_{\max }\left(A^{T}+A\right)\|x\|_{2}^{2}+3 \sqrt{3}\|x\|_{2}^{2}\right], \\
& \dot{\beta}(t)=-\lambda_{1} M \lambda_{\max }(P) \sqrt{3}\left[3|\beta| M+2\left\|\beta q\left(\frac{x}{\beta}\right)\right\| \operatorname{sign}(\beta)+\beta\|K\| \hat{\varepsilon} .\right.
\end{aligned}
$$

where the real number $\lambda_{0}>0$ and $\lambda_{1}>0$ are two designing constants. The quantization error bound $\varepsilon$ is unknown due to the quantization noise in practical applications, on this premise, $\varepsilon$ is estimated by using the suitable adaptive law in this paper. Let $\hat{\varepsilon}=\hat{\varepsilon}(t)$ denotes the estimated value of $\varepsilon$. The estimated error is $\tilde{\varepsilon}=\varepsilon-\hat{\varepsilon}$. By the united effect of the controller (10) and the quantizer parameter update laws (12), the adaptive law of $\hat{\varepsilon}$ is proposed as below.

$$
\begin{aligned}
& \dot{\hat{\varepsilon}}=2 \sqrt{3} \lambda_{2}|\beta| M \lambda_{\max }(P)\|K\|, \text { when }\|x\| \leq|\beta| M \\
& \dot{\hat{\varepsilon}}=0 \text {, when }\|x\|>|\beta| M .
\end{aligned}
$$

where the $\lambda_{2}>0$ is a designing constant.

Theorem 1 If Assumption 1is satisfied, the controller (10), associated with the parameter update laws (12) and (13), can stabilize the chaotic control system (7) asymptotically.

Proof The proof of Theorem 1 can be divided into two cases as follows.

Case $1\|x\|>|\beta| M$

In this case, it is proved that the expanded state $z=\left(x^{T}, \beta, \hat{\varepsilon}\right)^{T}$ of system (3) can enter the compact set $D=\left\{z|\|x\| \leq| \beta \mid M, z \in R^{5}\right\}$ by using the controller (10) and the parameter update laws (12) and (13). Let the sliding surface be noted as $s=0$, where $s=s\left(x^{T}, \beta, \tilde{\varepsilon}\right)^{T}=\|x\|^{2}-\beta^{2} M^{2}+0.5 \tilde{\varepsilon}^{2}$. Obviously, $s>0$ when $\|x\|>|\beta| M$. From inequality (3), it can get easily that

$$
\|F(x)\| \leq \sqrt{0+0+f^{2}\left(x_{1}\right)} \leq \frac{3}{2}\|x\| .
$$

Suppose $V=\frac{1}{2} s^{2}$, then $V$ is a positive definite function about $s$. The derivative of $V$ about $t$ is obtained as below,

$$
\begin{aligned}
\dot{V}(t) & =s\left(\dot{x}^{T} x+x^{T} \dot{x}-2 \beta M^{2} \dot{\beta}+\tilde{\varepsilon} \tilde{\tilde{\varepsilon}}\right) \\
& \leq s\left(\lambda_{\max }\left(A^{T}+A\right)\|x\|^{2}+2\|F(x)\|\|x\|-2 \beta M^{2} \dot{\beta}\right) \\
& =-\delta \mathrm{s}
\end{aligned}
$$

By inequality (15) and the result of [24], it is apparently seen that the expanded system state $z=\left(x^{T}, \beta, \hat{\varepsilon}\right)^{T}$ can enter the sliding surface $s=0$ in a finite time. Note that $\{z \mid s=0\} \subset D$, Theorem 1 in Case 1 is proved.

Case $2\|x\| \leq|\beta| M$
By using the conditions in the quantizer (4a), it is obtained

$$
\left\|F(x)-F\left(\beta q\left(\frac{x}{\beta}\right)\right)\right\| \leq \frac{3}{2}\left(\|x\|+\left\|\beta q\left(\frac{x}{\beta}\right)\right\|\right)
$$

By using the formulae (1), (12), (13) and (16), the derivative of the positive definite function $V(x)=V\left(x^{T}, \beta, \tilde{\varepsilon}\right)^{T}=x^{T} P x+\frac{1}{2} \lambda_{1}^{-1} \beta^{2}+\frac{1}{2} \lambda_{2}^{-1} \tilde{\varepsilon}^{2}$ respect to $t$ is obtained as follows.

$$
\begin{aligned}
\dot{V}(t) & =2 x^{T} P \dot{x}+\lambda_{1}^{-1} \beta \dot{\beta}+\lambda_{2}^{-1} \tilde{\tilde{\varepsilon}} \dot{\tilde{\varepsilon}}, \\
& \leq-x^{T} Q x+3\|x\| \lambda_{\max }(P)\left(\|x\|+\left\|\beta q\left(\frac{x}{\beta}\right)\right\|\right) \\
& +2 \sqrt{3}\|x\| \lambda_{\max }(P) \mid \beta\|\| K \| \varepsilon+\lambda_{1}^{-1} \beta \dot{\beta}+\lambda_{2}^{-1} \tilde{\varepsilon} \tilde{\tilde{\varepsilon}}, \\
= & -x^{T} Q x .
\end{aligned}
$$

The inequality (17) indicates that the expanded state $z$ is bounded, namely, the system state $x$ is bounded. By considering (12), (13) and (17), we can get that $\dot{x}(t)$ is also bounded in Case 2. Finally, by using the principle of Barbalat Lemma,[24] $\lim _{t \rightarrow \infty} x \rightarrow 0$ is obtained . Theorem 1 is completely proved with Case 1 and Case 2 .

\section{NUMERICAL SIMULATION EXAMPLES}

Because of the limitation length of the paper, only one chaotic systems from (1), in which the nonlinear term $f(x)$ takes the form $f(x)=f_{4}(x)$, is chosen for numerical simulation in this paper. Consider $a=0.5$ and $b=5$, and choose initial state $(1,0,-1)$ and $k_{1}=7, k_{2}=1, k_{3}=3$. By using quantizer (5) and controller (8), the simulation result shows that the controller (8), with quantizer (5), will lead controlled system (7) instability. Figure 1 shows the instability of the controlled system (7) with $f(x)=f_{4}(x)$. The closed-loop system (6) is globally asymptotically stable by a nonlinear feedback controller (10), by using a time varying quantizer as (6) in which the update law of $\beta(t)$ defined in (12) and the adaptive law of $\hat{\varepsilon}$ is (13). The illustration is showed in figure 2 .

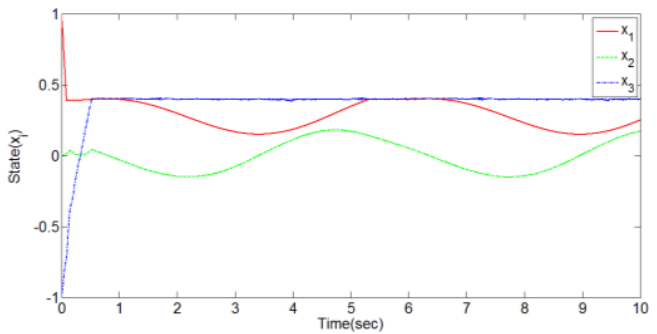

FIGURE 1. NUMERICAL SIMULATION OF STABILIZATION FOR CHAOS VIA THE NONLINEAR CONTROLLER (8) WITH THE QUANTIZER (5); THE INITIAL STATE IS $X 0=(1,0,-1)$, AND THE PARAMETERS ARE $a=0.5, b=5, k_{1}=7, k_{2}=1$ AND $k_{3}=3$. 


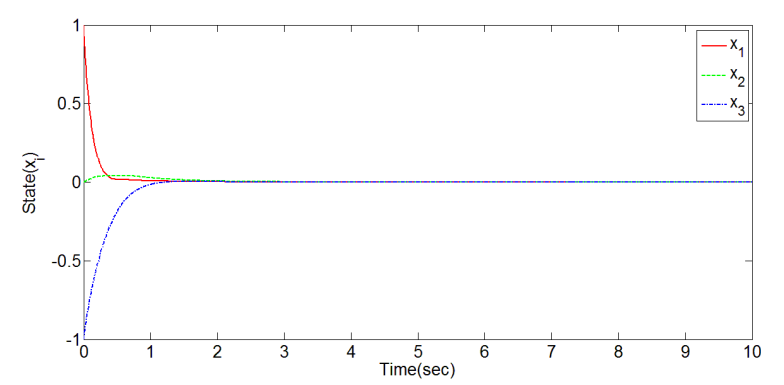

FIGURE I. NUMERICAL SIMULATION OF STABILIZATION FOR CHAOS VIA THE NONLINEAR CONTROLLER (10) WITH THE QUANTIZER (6); THE INITIAL STATE IS X0 $=(1,0,-1)$, AND THE

PARAMETERS ARE $a=0.5, b=5, k_{1}=7, k_{2}=1$ AND $k_{3}=3$.

\section{CONCLUSION}

The problem of stabilizing a class of chaotic systems, in which the nonlinear terms are monotonically increasing odd functions with the range $[-1,1]$, with state quantization measurements has been discussed. A nonlinear controller, associating with an adaptive quantizer which has two updated laws, is derived by the guide of the proposed method in this paper. it can ensure that the state variables of the controlled systems converge asymptotically to the origin.

\section{ACKNOWLEDGEMENTS}

The authors thank the financial supports of National Science Foundation of China (No. 61273219; 61305098); The Guangdong Natural Science Foundation (No. S2013010015768); the Project Program of KLGHEI (No. 2013CXZDA015); the Specialized Research Fund for the Doctoral Program of Higher Education of China (No. 20134420110003).

\section{REFERENCES}

[1] E. Ott, C. Grebogi, and J. A. Yorke, Physical Review Letters 64 (1990) 1196.

[2] T. Shinbrot, C. Grebogi, E. Ott and J. A. Yorke, Nature, 263 (1993) 411.

[3] H. T. Yau, C. O. K. Chen and C. L. Chen, International Journal Of Bifurcation And Chaos 10 (2000) 1139.

[4] X. Liu, Nonlinear Analysis-theory Methods \& Applications 47 (2001) 1081.

[5] D. Huang, Physical Review Letters 93 (2004) 214101.1.

[6] J. Huang, C. Li and X. He, International Journal of Control Automation and Systems 11 (2013) 643.

[7] S. H. Yu, H. S. Kang, Y. T. Kim, C. H. Hyun and M. Park, International Journal of Control Automation and Systems 12 (2014) 188.

[8] Y. Li, W. K. S. Tang and G. Chen, International Journal of Bifurcation And Chaos 15 (2005) 3367.

[9] M. Sun, L. Tian, S. Jiang and J. Xu, Chaos, Solitons \& Fractals 32 (2007) 1725.

[10] W. Yu, Physical Letters A 374 (2010) 3021.

[11] R. Zhang and S. Yang, Nonlinear Dynamics 68 (2012) 45.

[12] Z. Zhang, K. T. Chau and Z. Wang, IEEE Transactions on Vehicular Technology 62 (2013) 118.

[13] D. F. Delchamps, IEEE Transactions on Automatic Control 35 (1990) 916.

[14] M. Fu and L. Xie, IEEE Transactions on Automatic Control 54 (2009) 1165 .
[15] T. Ushio and K. Hirai, International Journal of Non-linear Mechanics 20 (1985) 493.

[16] D. Liberzon and J. P. Hespanha, IEEE Transactions on Automatic Control 50 (2005) 910.

[17] D. Liberzon, IEEE Transactions on Automatic Control 51 (2006) 1190.

[18] B. C. Zheng and G. H. Yang, International Journal of Robust and Nonlinear Control 24 (2014) 228

[19] Y. H. Wang, Y. H. Fan, Q. Y. Wang and Y. Zhang, Communications in Theoretical Physics 57 (2012) 808.

[20] J. X. Zhang, W. S Tang, Nonlinear Dynamics 58 (2009) 675.

[21] S.K. Kenue, Proceeding SPIE 1608(1992) 450.

[22] J.X. Zhang, W.S. Tang, Y. Xu, Acta Physica Sinica 57(2008), 6799 (in Chinese).

[23] J. Lü, G. Chen, X. Yu, H. Leung, IEEE Transactions on Circuits System I 51(2004) 2476.

[24] J. J. E. Slotine and W. P. Li, Applied nonlinear control, Prentice hall, Englewood Cliffs (1991). 